\section{Structured illumination enhances resolution and contrast in thick tissue fluorescence imaging}

\author{
Amaan Mazhar, ${ }^{\mathrm{a}, \mathrm{b}}$ David J. Cuccia, ${ }^{\mathrm{c}}$ Sylvain Gioux, ${ }^{\mathrm{d}, \mathrm{e}}$ \\ Anthony J. Durkin, ${ }^{\mathrm{b}}$ John V. Frangioni, ${ }^{\mathrm{e}}$ and \\ Bruce J. Tromberg ${ }^{a, b}, *$ \\ anniversity of California, Irvine, Department of Biomedical \\ Engineering, Irvine, California 92612 \\ ${ }^{b}$ University of California, Irvine, Beckman Laser Institute \\ and Medical Clinic, Laser Microbeam and Medical \\ Program, Irvine, California 92612 \\ cModulated Imaging, Inc., Technology Incubator Office, \\ Irvine, California 92612 \\ dBoston University, Department of Biomedical Engineering, \\ Boston, Massachusetts 02215 \\ ${ }^{\mathrm{e}}$ Beth Israel Deaconess Medical Center, Department of \\ Medicine, Division of Hematology/Oncology, \\ Boston, Massachusetts 02215
}

\begin{abstract}
We introduce a noncontact imaging method utilizing multifrequency structured illumination for improving lateral and axial resolution and contrast of fluorescent molecular probes in thick, multiple-scattering tissue phantoms. The method can be implemented rapidly using a spatial light modulator and a simple image demodulation scheme similar to structured light microscopy in the diffraction regime. However, imaging is performed in the multiple-scattering regime utilizing spatially modulated scalar photon density waves. We demonstrate that by increasing the structured light spatial frequency, fluorescence from deeper structures is suppressed and signals from more superficial objects enhanced. By measuring the spatial frequency dependence of fluorescence, background can be reduced by localizing the signal to a buried fluorescent object. Overall, signal-to-background ratio (SBR) and resolution improvements are dependent on spatial frequency and object depth/dimension with as much as sevenfold improvement in SBR and 33\% improvement in resolution for $\sim 1-\mathrm{mm}$ objects buried $3 \mathrm{~mm}$ below the surface in tissue-like media with fluorescent background. () 2010 Society of Photo-Optical Instrumentation Engineers. [DOI: 10.1117/1.3299321]
\end{abstract}

Keywords: diffuse optics; near infrared; photon migration; spatial modulation.

Paper 09396LR received Sep. 8, 2009; revised manuscript received Dec. 3, 2009; accepted for publication Dec. 4, 2009; published online Mar. 2, 2010.

There is considerable interest in the development of in vivo near-infrared (NIR) fluorescence imaging and tomography for clinical and preclinical research. ${ }^{1,2}$ In the NIR, photon penetration is maximized due to reduced light scattering and relatively low absorption of intrinsic tissue chromophores such as water, hemoglobin, and lipid.

*Address all correspondence to: Bruce J. Tromberg. Tel: 949-824-8367; Fax: 949-824-6969; E-mail: bjtrombe@uci.edu
NIR excitation and subsequent emission can significantly improve imaging depth compared to visible fluorescent probes. Despite this advantage, accurate mapping of subsurface (i.e., $\mathrm{mm}$ to $\mathrm{cm}$ ) fluorescence with high spatial resolution remains a critical challenge. This is due to the fact that lateral and axial localization of fluorescence is "blurred" by multiple light scattering. In order to address this limitation, imaging systems that utilize multiple detectors, source scanning, and time/frequency domain techniques have been successfully implemented based on diffuse optical tomography principles. $^{3-5}$

In this work, we demonstrate a simple alternative nonscanning method using multifrequency structured illumination patterns to improve axial and transverse resolution of buried fluorescent inhomogeneities. Our approach utilizes similar principles to technologies employing structured illumination developed for the diffraction regime. ${ }^{6,7}$ However, in this case, lateral and axial resolution improvements are obtained from thick, turbid materials using principles of scalar photon density waves propagating in the diffusion regime. ${ }^{8-10}$

A spatial light modulator is introduced at the source, and a number of sinusoidal illumination patterns with different spatial frequencies, $k$, are projected over a tissue sample at three offset phases (typically $0 \mathrm{deg}, 120 \mathrm{deg}$, and $240 \mathrm{deg}$ ). During data analysis, the images at each phase, $I_{1,2,3}$, are demodulated using Eq. (1) in order to analyze the AC component of the remitted light. This concept has also been applied by Neil et al. for the purposes of optical sectioning microscopy. ${ }^{11}$

$$
\left.M_{A C}\left(x_{i}\right)\right|_{f_{x i}}=\frac{2^{1 / 2}}{3}\left[\left(I_{1}-I_{2}\right)^{2}+\left(I_{2}-I_{3}\right)^{2}+\left(I_{3}-I_{1}\right)^{2}\right]^{1 / 2} \text {. }
$$

In the spatial frequency domain, the reflected AC component of the spatially modulated waves can be used to characterize the modulation transfer function (MTF) of the sample. ${ }^{8,9}$ We now extend this concept to fluorescence imaging where structured light illumination specifically impacts the excitation fluence rate. A forward fluence model for spatially modulated light—-specifically, sinusoidal waves — can be adapted from a 1-D second-order Helmholtz equation in order to calculate fluence rate $(\varphi)$ as a function of depth, $z:^{8}$

$$
\frac{d^{2}}{d z^{2}} \varphi_{0}(z)-\mu_{e f f}^{\prime 2} \varphi_{0}(z)=-3 \mu_{t r} q_{0}(z),
$$

In these equations, $q$ is the source, $\mu_{t r}=\left(\mu_{a}+\mu_{s}^{\prime}\right)$ is the transport coefficient, $\mu_{a}$ is the absorption coefficient, $\mu_{s}^{\prime}$ $=\mu_{s}(1-g)$ is the reduced scattering coefficient, $g$ is the mean cosine of the scattering angle $(\langle\cos \theta\rangle), k$ is the spatial frequency of the illumination pattern, $\mu_{e f f}=\left[3 \mu_{a} \mu_{t r}\right]^{1 / 2}$, and $\mu_{\text {eff }}^{\prime}=\left[\mu_{e f f}^{2}+k^{2}\right]^{1 / 2}$. The solution for this equation is:

$$
\varphi_{0}(z)=\frac{3 P_{0} a^{\prime}}{\mu_{e f f}^{\prime 2} / \mu_{t r}^{2}-1} \exp \left(-\mu_{t r} z\right)+C \exp \left(-\mu_{e f f}^{\prime} z\right),
$$

where $a^{\prime}$ is the reduced albedo $\left(\mu_{s}^{\prime} / \mu_{t r}^{\prime}\right)$, is the incident optical power, and $C$ is a constant determined by the choice of a boundary condition. A normalized fluence rate profile for

1083-3668/2010/15(1)/010506/3/\$25.00 @ 2010 SPIE 


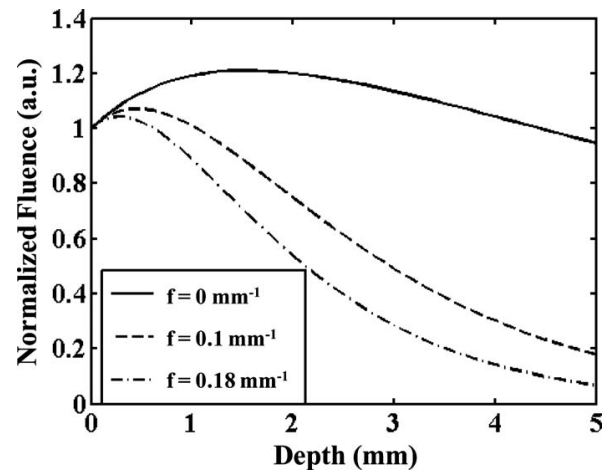

Fig. 1 Fluence profiles for spatially modulated illumination at three different spatial frequencies demonstrate relative sensitivity at different depths.

three frequencies (Fig. 1) is plotted as a function of depth for a given set of optical properties $\left(\mu_{a}=0.005 \mathrm{~mm}^{-1}, \mu_{s}^{\prime}\right.$ $=0.8 \mathrm{~mm}^{-1}$ ). Tissue behaves as a low-pass spatial filter as the normalized excitation fluence rate decays more rapidly at higher illumination frequencies, allowing depth-sensitive excitation of buried fluorescent inclusions.

For experiments, structured NIR light is projected onto the sample using a modified digital light projector (NEC HT1000). The micromirrors allow generation of desired patterns as shown in other applications. ${ }^{7,9}$ For fluorescence imaging, the color's filter wheel was removed and an interference bandpass filter $(\lambda=660 \mathrm{~nm}, \delta=10 \mathrm{~nm}$ FWHM) was placed at the source, and a second bandpass filter $(\lambda$ $=720 \mathrm{~nm}, \delta=10 \mathrm{~nm}$ FWHM) was placed at the CCD camera (Roper QuantEM: 512SC). Cross-linear polarizers were also placed at the source and detector to eliminate specular signals in the case of reflectance imaging, although they are not necessary for fluorescence.

In order to explore axial and lateral resolution, respectively, imaging was performed on two polydimethylsiloxane (PDMS) phantoms ${ }^{12}$ with embedded channels and tissue-like optical properties $\left(\mu_{a}=0.005 \mathrm{~mm}^{-1}, \mu_{s}^{\prime}=0.8 \mathrm{~mm}^{-1}\right)$. Phantom 1 contained four 1-mm-diam tube inclusions, each separated laterally by $1 \mathrm{~cm}$ at depths of $1 \mathrm{~mm}, 2 \mathrm{~mm}, 3 \mathrm{~mm}$, and $4 \mathrm{~mm}$, respectively [Video 1(a)]. The fluorophore injected into the phantom tubes consisted of a mixture of $250 \mathrm{nM}$ IRDye 700DX dye (LyCOR, Omaha, Nebraska) dissolved in a solution of $0.8 \%$ Intralipid diluted with deionized water in order to match background scattering. Imaging was performed on each phantom using 16 evenly spaced illumination frequencies ranging from $0 \mathrm{~mm}^{-1}$ to $0.3 \mathrm{~mm}^{-1}$ [Video 1(b)]. Sinusoidal illumination patterns were projected with frequency, $k$, oriented in the horizontal $(x)$ direction. Three phases ( $0 \mathrm{deg}, 120 \mathrm{deg}, 240 \mathrm{deg})$ were acquired for each frequency at constant exposure times (typically $500 \mathrm{~ms}$ ), and images were demodulated using Eq. (1). Demodulated images were calibrated by dividing by a reference unstructured planar (i.e., $0 \mathrm{~mm}^{-1}$ ) illumination image obtained from a gelatin phantom with evenly distributed fluorophore [Video 1(c)]. Video 1 illustrates the entire image acquisition and demodulation process and as predicted by the forward fluence model, the fluorescence contribution from deeper tubes diminishes as the illumination frequency increases due to attenuation of (a) Phantom

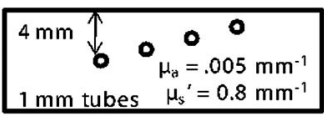

(c) $\mathrm{F}_{\mathrm{d}}, \mathrm{f}=0.3 \mathrm{~mm}^{-1}$

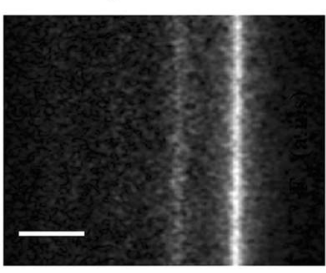

(b) Projection, $f=0.3 \mathrm{~mm}^{-1}$

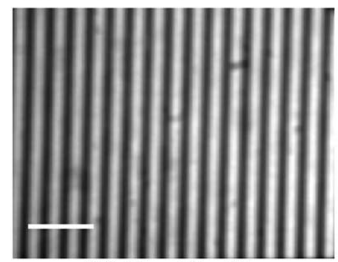

(d) Line Profile

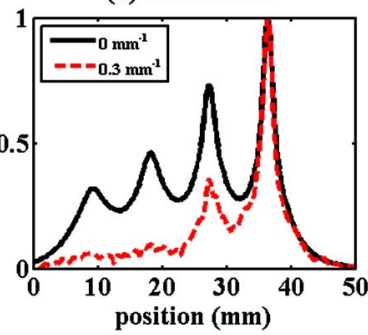

Video 1 Depth sensitive imaging with multifrequency structured illumination. (a) Phantom 1 diagram. (b) Projection pattern. (c) Demodulated and calibrated fluorescence map. (d) Normalized horizontal line profiles highlight frequency-dependent depth sensitivity due to suppression of signal from deep tubes at high frequencies. (QuickTime, 1.46 MB). [URL: http://dx.doi.org/10.1117/1.3299321.1].

longer path length excitation photons. ${ }^{9}$ Horizontal line profiles of these images highlight the frequency-dependent depth sensitivity, revealing only the most superficial tubes at the higher spatial frequency [Video 1(d)].

Imaging was performed in the same manner on Phantom 2, consisting of two 2-mm-diam tubes separated by $5 \mathrm{~mm}$ and positioned $4 \mathrm{~mm}$ below the surface [Fig. 2(a)]. Calibrated and demodulated fluorescence images [Fig. 2(b)] illustrate that planar unstructured illumination $\left(0 \mathrm{~mm}^{-1}\right)$ yields an image of a single tube at the surface, while an increase in spatial frequency $\left(0.1 \mathrm{~mm}^{-1}\right)$ produces two optimally resolved tubes [Fig. 2(c)]. The resolution improvement seen at $0.1 \mathrm{~mm}^{-1}$ is lost at $0.18 \mathrm{~mm}^{-1}$ due to suppression of long path length excitation photons resulting in an interrogation region predominantly above the buried tubes. Thus, although increasing spa-

(a)

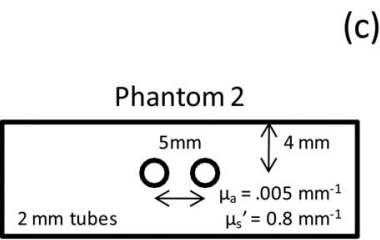

(b)

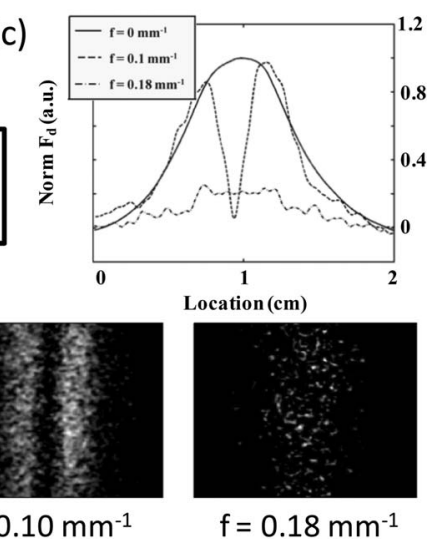

Fig. 2 Improved transverse resolution with multifrequency structured illumination. (a) Phantom 2 diagram. (b) Demodulated and calibrated fluorescence maps at three spatial frequencies. (c) Horizontal line profiles at each spatial frequency confirm improved transverse resolution by resolving two tubes at optimal spatial frequency. 


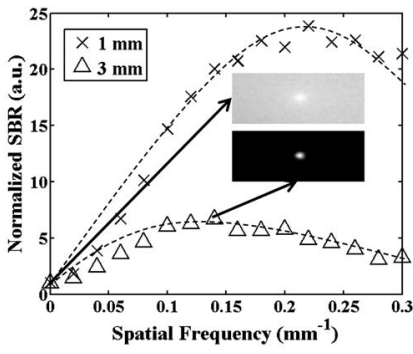

(a)

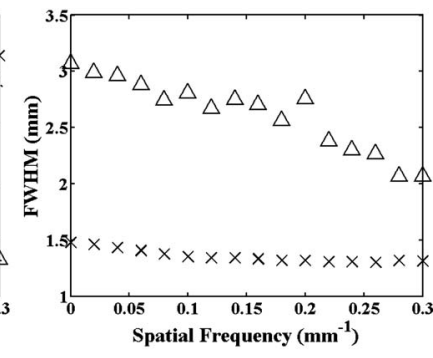

(b)
Fig. 3 Improved signal-to-background ratio (SBR) and resolution. A 1 -mm fluorescent bead (incubated in $10 \mu \mathrm{M}$ IR 700 solution) was buried $1 \mathrm{~mm}(\times)$ and $3 \mathrm{~mm}(\triangle)$ below the surface of a 100-nM fluorescent phantom and imaged at 16 spatial frequencies. In general, the (a) SBR improved to a point as spatial frequency increased and (b) the full width at half maximum (FWHM) decreased as spatial frequency increased. Inset pictures represent images of the bead buried $3 \mathrm{~mm}$ below the surface at a spatial frequency of $0 \mathrm{~mm}^{-1}$ (top) and optimized frequency of $0.14 \mathrm{~mm}^{-1}$ (bottom).

tial frequency clearly improves lateral resolution, this effect is convolved with the insensitivity of high-frequency waves to deeper objects.

Last, a third set of phantoms was prepared using the protocol described by De Grand et al. ${ }^{13}$ to explore the trade-off between contrast and resolution. One-mm-diam polystyrene beads incubated in a $10-\mu \mathrm{M}$ IR700 solution for $24 \mathrm{~h}$ were buried 1 and $3 \mathrm{~mm}$ below the surface of homogenous fluorescent gelatin phantoms with 100-nM background fluorescence and tissue-like optical properties $\left(\mu_{a}=.01 \mathrm{~mm}^{-1}, \quad \mu_{s}^{\prime}\right.$ $\left.=0.8 \mathrm{~mm}^{-1}\right)$. Phantoms were imaged and calibrated as described earlier. The normalized signal-to-background ratio [SBR, Fig. 3(a)] was calculated by taking the ratio of the average count of the pixels that represented the inclusion full width at half maximum (FWHM) and the background regions at each spatial frequency. The bead FWHM [Fig. 3(b)] was calculated from normalized horizontal line profiles.

Figure 3(a) shows that SBR is maximized at illumination frequency of $0.22 \mathrm{~mm}^{-1}$ with $\sim 2400 \%$ improvement for the superficial structure (versus $0 \mathrm{~mm}^{-1}$ ) and at $0.14 \mathrm{~mm}^{-1}$ with $\sim 700 \%$ improvement for the deeper structure. Resolution improvements are limited by SBR and multiple scattering. Figure $3(\mathrm{~b})$ shows that resolution of the beads improves with increasing illumination frequency by "gating" of long path length photons but remains greater than $1 \mathrm{~mm}$ due to some scattering. At $0.3 \mathrm{~mm}^{-1}$, the apparent diameter for the deeper bead is $\sim 2 \mathrm{~mm}$, a $\sim 33 \%$ improvement in resolution (versus $\left.0 \mathrm{~mm}^{-1}\right)$. The 1 -mm-deep inclusion appears to be $\sim 1.3 \mathrm{~mm}$, a $\sim 13 \%$ improvement. Thus, resolution and contrast of subsurface fluorescent structures can be improved via multifrequency illumination. The optimal frequency depends on the depth of the target structure and the background optical properties (i.e., absorption, scattering, and fluorescence).

We have demonstrated a method to improve lateral and axial resolution in fluorescence imaging of turbid media using multifrequency structured light. Image formation is achieved using a simple demodulation scheme that effectively provides spatial frequency "gating" due to the frequency-dependent penetration depth of the excitation fluence. This method improves localization and SBR compared to planar imaging techniques and is amenable to the development of further tomographic algorithms. ${ }^{14}$ Potential applications of this approach include imaging of small animal models as well as imaging subsurface structures in clinical settings.

\section{Acknowledgments}

This research was made possible by the Laser Microbeam and Medical Program (LAMMP), an NIH Biomedical Technology Resource, Grant No. P41-RR01192; the National Cancer Institute, Grant No. R21-CA129758; the Beckman Foundation; and the Military Photomedicine Program, AFOSR Grant No. FA9550-08-1-0384.

\section{References}

1. J. V. Frangioni, "In vivo near-infrared fluorescence imaging," Curr. Opin. Chem. Biol. 7, 626-634 (2003).

2. V. Ntziachristos, C. Bremer, and R. Weissleder, "Fluorescence imaging with near-infrared light: new technological advances that enable in vivo molecular imaging," Eur. Radiol. 13(1), 195-208 (2003).

3. E. E. Graves, J. Ripoll, R. Weissleder, and V. Ntziachristos, "A submillimeter resolution fluorescence molecular imaging system for small animal imaging," Med. Phys. 30(5), 901-911 (2003).

4. A. J. Chaudhari, F. Darvas, J. R. Bading, R. A. Moats, P. S. Conti, D. J. Smith, S. R. Cherry, and R. M. Leahy, "Hyperspectral and multispectral bioluminescence optical tomography for small animal imaging," Phys. Med. Biol. 50(23), 5421-5441 (2005).

5. V. Ntziachristos, J. Ripoll, L. V. Wang, and R. Weissleder, "Looking and listening to light: the evolution of whole-body photonic imaging," Nat. Biotechnol. 23(3), 313-320 (2005).

6. M. G. Gustafsson, "Nonlinear structured-illumination microscopy: wide-field fluorescence imaging with theoretically unlimited resolution," Proc. Natl. Acad. Sci. U.S.A. 102(37), 13081-13086 (2005).

7. S. Delica and C. M. Blanca, "Wide-field depth-sectioning fluorescence microscopy using projector-generated patterned illumination," Appl. Opt. 46(29), 7237-7243 (2007).

8. D. J. Cuccia, F. Bevilacqua, A. J. Durkin, and B. J. Tromberg, "Quantitation and mapping of tissue optical properties using modulated imaging," J. Biomed. Opt. 14(2), 024012 (2009).

9. D. J. Cuccia, F. Bevilacqua, A. J. Durkin, and B. J. Tromberg, "Modulated imaging: quantitative analysis and tomography of turbid media in the spatial-frequency domain," Opt. Lett. 30(11), 13541356 (2005).

10. A. Bassi, C. D’Andrea, G. Valentini, R. Cubeddu, and S. Arridge, "Temporal propagation of spatial information in turbid media," Opt. Lett. 33(23), 2836-2838 (2008).

11. M. A. Neil, R. Juskaitis, and T. Wilson, "Method of obtaining optical sectioning by using structured light in a conventional microscope," Opt. Lett. 22(24), 1905-1907 (1997).

12. F. Ayers, A. Grant, D. Kuo, D. J. Cuccia, and A. J. Durkin, "Fabrication and characterization of silicone-based tissue phantoms with tunable optical properties in the visible and near-infrared domain," Proc. SPIE 6870, 687007 (2008).

13. A. M. De Grand, S. J. Lomnes, D. S. Lee, M. Pietrzykowski, S. Ohnishi, T. G. Morgan, A. Gogbashian, R. G. Laurence, and J. V. Frangioni, "Tissue-like phantoms for near-infrared fluorescence imaging system assessment and the training of surgeons," J. Biomed. Opt. 11(1), 014007 (2006).

14. S. D. Konecky, A. Mazhar, D. Cuccia, A. J. Durkin, J. C. Schotland, and B. J. Tromberg, "Quantitative optical tomography of subsurface heterogeneities using spatially modulated structured light," Opt. Express 17(17), 14780-14790 (2009). 